Preprint: Sangeetha Periasamy, Sasirekha Venkidusamy, Ragavendran Venkatesan, Jeyanthinath Mayandi, Joshua Pearce, Josefine Helene Selj, Ramakrishnan Veerabahu. Micro-Raman Scattering of Nanoscale Silicon in Amorphous and Porous Silicon. Zeitschrift für Physikalische Chemie, 231(9), pp. 1585-1598 (2017). DOI: https://doi.org/10.1515/zpch-2016-0961

\title{
Micro-Raman Scattering of Nanoscale Silicon in Amorphous and Porous Silicon
}

\author{
P.Sangeetha $^{1}$, V.Sasirekha ${ }^{2}$, J. Mayandi ${ }^{3,4}$, J. M. Pearce, ${ }^{4}$ and V. Ramakrishnan ${ }^{1}$ \\ ${ }^{1}$ Department of Laser Studies, School of Physics, Madurai Kamaraj University, Madurai - 625 \\ 021, India. \\ ${ }^{2}$ Department of Physics, Avinasilingam University, Coimbatore-641 043, India. \\ ${ }^{3}$ Department of Materials Science, School of Chemistry, Madurai Kamaraj University, Madurai- \\ 625 021, India. \\ ${ }^{4}$ Department of Materials Science \& Engineering, Michigan Technological University, USA \\ ${ }^{5}$ Department of Electrical \& Computer Engineering, Michigan Technological University, USA
}

\begin{abstract}
Thesize effect of nanoscale silicon in both amorphous and porous silicon was investigated with micro-Raman spectroscopy. Silicon nanostructers in amorphous silicon were deposited on quartz substrates by plasma enhanced chemical vapor deposition (PECVD) with deposition powers of 15, 30 and 50W. Micro-Raman spectra of the nanostructured silicon show the $\mathrm{T}_{2 \mathrm{~g}}$ Raman active mode shifting from the $521 \mathrm{~cm}^{-1}$ crystalline Si Raman line to 494, 499 and $504 \mathrm{~cm}^{-1}$ as deposition power increased. Large Raman mode shifts, up to $27 \mathrm{~cm}^{-1}$ and broadening up to $23 \mathrm{~cm}^{-1}$ of the $\mathrm{T}_{2 \mathrm{~g}}$ Raman-active mode is attributed to a phonon confinement effect which \{did what with power\}. The analysis of micro-Raman scattering data is useful to understand the role of deposition condition of the silicon sample. In addition, enhanced micro-Raman scattering intensity of porous silicon prepared using double cell electrochemical etching through various current densities such as 10,50 and $125 \mathrm{~mA} / \mathrm{cm}^{2}$ has also been investigated. The effect of phonon confinement on the nanoscale porous silicon has been quantified. The relationship between Raman shift and stress on the porous silicon has been evaluated.
\end{abstract}

\section{Introduction}

Technological interest in silicon ( $\mathrm{Si}$ ) nanostructures, with sizes of about 2-5 $\mathrm{nm}$ that display quantum size effects, arises from their potential applications in silicon based optoelectronics [4]. Si-based nanostructures are fundamentally different from those of the bulk crystalline silicon (c-Si) material and make nanoscale silicon attractive for use in a variety of important technological applications including thin-film transistors (TFTs), solar photovoltaic (PV) cells, peripheral circuits of liquid crystal displays (LCDs), and electrodes in Si integrated circuits [1,2]. For example, the inclusion of nanocrystals in the p-layer of an hydrogenated amorphous silicon (a-Si:H) solar PV cell was found to have optimal open circuit voltage [ ] and inclusion of nanocrystals in the i-layer was found to increase a-Si:H PV stability []. In addition to that material of porous silicon, nanometer sized silicon crystallites has become the subject of great interest. This is because they exhibit new quantum phenomena and have favorable 
Preprint: Sangeetha Periasamy, Sasirekha Venkidusamy, Ragavendran Venkatesan, Jeyanthinath Mayandi, Joshua Pearce, Josefine Helene Selj,

Ramakrishnan Veerabahu. Micro-Raman Scattering of Nanoscale Silicon in Amorphous and Porous Silicon. Zeitschrift für Physikalische

Chemie, 231(9), pp. 1585-1598 (2017). DOI: https://doi.org/10.1515/zpch-2016-0961

applications in optoelectronic devices [3]. During the recent years, nanostructured silicon is a promising key material to establish a Si-based photonics [refs] needed.

The most commonly used preparation technique, silicon deposited on quartz, is plasma enhanced chemical vapor deposition (PECVD). The structural parameters of the precipitated silicon nanostructures depend strongly on the deposition conditions. Porous silicon is commonly made by electrochemical etching of $\mathrm{Si}$ in an electrolyte containing hydrofluoric acid (HF). The nanostructure is very sensitive to etching parameters such as current density, electrolyte composition, temperature and substrate doping [5].

Raman spectroscopy is recognized as a powerful technique for the characterization of silicon nanostructures [refs]. Micro-Raman spectroscopy allows identification of the material and yields information about phonon frequencies, electron-phonon interaction, impurity content, composition, crystal structure, crystal orientation and mechanical strain [6]. This study reports on a micro-Raman investigation of nanoscale silicon in amorphous silicon deposited on quartz and porous silicon fabricated by electrochemical etching of crystal silicon. Both the influence of rf power deposition and current density on crystallinity and the effect of compressive stress on porous silicon are analyzed. A phonon confinement model is provided as theoretical basis for understanding the nature of nanostructured silicon and that the model is validated with the experimental results.

\section{Experimental Methods}

\subsection{Material Synthesis}

Amorphous silicon was deposited on quartz substrates by PECVD at a deposition temperature $650^{\circ} \mathrm{C}$ using precursor gases of silane $\left(\mathrm{SiH}_{4}\right)$, argon $(\mathrm{Ar})$ and hydrogen $\left(\mathrm{H}_{2}\right)$ with flow rate of 50, 30 and $20 \mathrm{sccm}$, respectively. The rf deposition power was $15 \mathrm{~W}, 30 \mathrm{~W}$ and 50W.

Porous silicon was prepared with a double cell electrochemical etching system using boron doped monocrystalline silicon (c-Si) (100) oriented wafer with a resistivity of 0.012-0.018 $\Omega \mathrm{cm}$. The electrolyte consisted of $40 \% \mathrm{HF}$ and $60 \%$ ethanol by volume. Three sets of samples were fabricated; the first etched at a current density of $10 \mathrm{~mA} / \mathrm{cm}^{2}$ for $30 \mathrm{~s}$, the second set etched at $50 \mathrm{~mA} / \mathrm{cm}^{2}$ for $15 \mathrm{~s}$ and the third set etched at $125 \mathrm{~mA} / \mathrm{cm}^{2}$ for $15 \mathrm{~s}$.

\subsection{Micro-Raman Spectroscopy}

The micro-Raman spectra were recorded from the top surface of all samples at room temperature using a HORIBA Jobin Yvon LabRam HR 800 spectrometer with a thermoelectrically cooled charge coupled detector (CCD) with a spectral resolution was $0.3 \mathrm{~cm}^{-1}$. The spectrograph contains holographic grating with 1800 grooves $\mathrm{mm}^{-1}$. The Calibration was performed using the $521 \mathrm{~cm}^{-1}$ band of a pure Si wafer. A $17 \mathrm{~mW} \mathrm{He-Ne} \mathrm{laser}(633 \mathrm{~nm})$ focused with a 100X objective to a $1 \mu \mathrm{m}$ spot diameter was used to excite the silicon.The Raman scattering spectra of silicon/quartz were measured from 400 to $600 \mathrm{~cm}^{-1}$ wavenumbers. All the measurements were performed in the geometry of back scattering. The Raman signatures of amorphous silicon and porous silicon were uniquely distinguishable for the two sets of samples 
Preprint: Sangeetha Periasamy, Sasirekha Venkidusamy, Ragavendran Venkatesan, Jeyanthinath Mayandi, Joshua Pearce, Josefine Helene Selj,

Ramakrishnan Veerabahu. Micro-Raman Scattering of Nanoscale Silicon in Amorphous and Porous Silicon. Zeitschrift für Physikalische

Chemie, 231(9), pp. 1585-1598 (2017). DOI: https://doi.org/10.1515/zpch-2016-0961

and they contained no traces of $\mathrm{SiO}_{2}$. Origin 8.0 was used to apply Lorentzian curve fits to the Raman active modes of the samples.

\section{Results and Discussion}

\subsection{Micro-Raman scattering analysis of Si/quartz}

Fig. 1 shows the micro-Raman spectra of amorphous silicon onquartz with various rf deposition powers. An intense Raman line at $521 \mathrm{~cm}^{-1}$ for a single crystal Si wafer is shown as reference. c-Si, which is cubic in nature, belongs to the crystal symmetry class $\mathrm{O}_{\mathrm{h}}$ and gives rise to a single triply degenerate Raman active $\mathrm{T}_{2 \mathrm{~g}}$ phonon mode around $521 \mathrm{~cm}^{-1}$. This band is due to the first-order longitudinal optical phonon mode (LO) [7]. As seen in the Fig. 1 the Raman peak at $504 \mathrm{~cm}^{-1}$ (50W) is corresponding to $\mathrm{Si}-\mathrm{Si}$ longitudinal optical (LO) phonon present in amorphous silicon. Also the broad Raman peaks at $494(15 \mathrm{~W})$ and $499 \mathrm{~cm}^{-1}(30 \mathrm{~W})$ are correlated to amorphous nature of the Si [8]. Though the spectra reveal an amorphous nature of the silicon, there is a possibility of small grains of crystalline or polycrystalline silicon. All other modes are not Raman active because of symmetry in the face-centered-cubic lattice. However, when the crystalline silicon grain size is as small as a few $\mathrm{nm}$, momentum conservation will be relaxed and Raman active modes will not be limited to the center of the Brillouin zone [9]. Thus, the frequency could shift from 504 to $494 \mathrm{~cm}^{-1}$. Below $1 \mathrm{~nm}$, a crystalline to amorphous transition occurs. In Fig. 1 Raman mode of Si/quartz reflects short range order and dependence of local bond angles on the phonon density of states.

A Lorentzian curve fit for the $\mathrm{T}_{2 \mathrm{~g}}$ Raman active mode of Si/quartz with various deposition powers is shown in Fig. 2. The area and FWHM of $\mathrm{T}_{2 \mathrm{~g}}$ phonon mode of silicon are calculated from the Lorentzian curve fit and are listed in Table 1. Fig. 3 indicates that with increasing deposition power the Raman peak position is shifted towards higher wavenumbers while the FWHM decreases.

The appearance of the broad bands indicates the amorphous silicon as the disorder in the surface layer of the silicon particles is attributed to the amorphous structure. With increasing deposition power, the area of the $\mathrm{T}_{2 \mathrm{~g}}$ phonon line of $\mathrm{Si} /$ quartz decreases due to grain boundaries. The $\mathrm{T}_{2 \mathrm{~g}}$ phonon line is shifted to lower wavenumbers (red shifted) by about 27 to $17 \mathrm{~cm}^{-1}$ from that of single crystalline silicon. This appreciable red shift is due to the amorphous and quantum confinement effect [10]. The Raman spectrum of amorphous silicon arises due to the presence of surface - like Si-Si shearing mode at the grain boundaries [11]. The intensity of the LO phonon mode at $494 \mathrm{~cm}^{-1}$ is high in comparison with that of phonon mode at $504 \mathrm{~cm}^{-1}$. These results indicate that the silicon structures are not totally crystallized even after increasing the rf power. Here, the force and stress are fully relaxed due to the expansion of the Si lattice. The effects of spatial confinement of phonons in small silicon particles have been studied theoretically and experimentally. Ding Wen-Ge et al. reported a size dependent shift $\Delta \omega$ of the silicon phonon mode with respect to that of crystalline silicon $\left(521 \mathrm{~cm}^{-1}\right)[12,10]$, providing the following relation: 
Preprint: Sangeetha Periasamy, Sasirekha Venkidusamy, Ragavendran Venkatesan, Jeyanthinath Mayandi, Joshua Pearce, Josefine Helene Selj, Ramakrishnan Veerabahu. Micro-Raman Scattering of Nanoscale Silicon in Amorphous and Porous Silicon. Zeitschrift für Physikalische Chemie, 231(9), pp. 1585-1598 (2017). DOI: https://doi.org/10.1515/zpch-2016-0961

$$
\Delta \omega(\mathrm{D}):=-\mathrm{A} \cdot \mathrm{H} \frac{\alpha}{\mathrm{D}]} \mathrm{H}^{\gamma} \longrightarrow
$$

Where $\Delta \omega(\mathrm{D})$ is the Raman shift in a nanostructures with diameter $\mathrm{D}, \alpha$ is the lattice constant of silicon $(0.543 \mathrm{~nm})$ and $A=47.41 \mathrm{~cm}^{-1}, \gamma=1.44$ are fit parameters that describe the phonon confinement in nanometric spheres of diameter D. The size of the Si nanocrystals is determined from the shift of the $\mathrm{T}_{2 \mathrm{~g}}$ optically active phonon mode. The result of applying equation 1 to $50 \mathrm{~W}$ sample shows that the size of the silicon nanocrystal is about $1 \mathrm{~nm}$. The decrease in the size of a silicon crystal in all direction is attributed to the confinement of the spatial wave function of optical phonon [13].

The large phonon softening (shift of the phonon mode) is also observed in fig. 1 and this feature is assumed to be the result of size related increase of disorder. The increase of disorder, produced by the small particle volume, results in breaking the wave-vector Raman scattering selection rule $(\mathrm{k}=0)$ and causes the softening of the phonon mode in the Raman spectra [14]. Hence, the phonon confinement model can be used for the calculation of the size of the silicon nanocrystals. Using this model the first order Raman intensity I $(\omega)$ is given by $[15,16,17]$

$$
\begin{aligned}
& \mathrm{I}(\omega)=\int_{0}^{1} \frac{e^{-\frac{\mathrm{qL} B^{2}}{4 \pi}} 4 \pi \mathrm{q}^{2}}{\left.(\omega-\omega(\mathrm{q}))^{2}+\frac{\Gamma_{0}}{2}\right]^{2}} d^{3} \mathrm{q} \longrightarrow \\
& \omega(q)=A-B q^{2}
\end{aligned}
$$

where $\omega(\mathrm{q})$ represents the phonon dispersion relation for an optical branch of crystallıne silicon and $\mathrm{a}$ is the lattice constant. Furthermore $\Gamma_{0}$ and $\omega$ are the natural linewidth and wavenumber of Raman line of the crystalline silicon, respectively and $\mathrm{L}$ is the crystalline grain size. Fig. 4 shows the calculated Raman spectra of silicon with various crystalline grain size (L). As can be seen in Fig. 4, for values of L below 150nm, the Raman band is shifted towards lower wavenumbers and broadened. Moreover, both band shift and broadening also increases as L decreases. Here the experimental and theoretical Raman $\mathrm{T}_{2 \mathrm{~g}}$ mode of silicon $(50 \mathrm{~W})$ is fit satisfactorily, when the crystalline grain size of silicon (50W) is $1 \mathrm{~nm}$.

The crystalline volume fraction can be calculated as follows [18]

$$
X_{c}=\frac{I_{c}}{I_{c}+0.8 I_{a}}
$$

Where $I_{c}$ and $I_{a}$ denoted the integrated intensities of the crystalline and amorphous peaks respectively. The crystalline fraction can be strongly correlated to the grain boundaries of the sample. The value of $X_{c}$ is $56 \%$ for silicon (50W) sample. This indicates that the structure order of Si-Si network in the film is not better due to its grain boundaries [7]. 
Preprint: Sangeetha Periasamy, Sasirekha Venkidusamy, Ragavendran Venkatesan, Jeyanthinath Mayandi, Joshua Pearce, Josefine Helene Selj,

Ramakrishnan Veerabahu. Micro-Raman Scattering of Nanoscale Silicon in Amorphous and Porous Silicon. Zeitschrift für Physikalische

Chemie, 231(9), pp. 1585-1598 (2017). DOI: https://doi.org/10.1515/zpch-2016-0961

\subsection{Micro-Raman scattering analysis of porous silicon/silicon}

Micro-Raman measurements of porous silicon were recorded for two regions of the film: one is dark circled area (porous silicon) and another in the substrate (crystalline silicon). Fig. 5 indicates the micro-Raman scattering of porous silicon with different porosity. Here, the Raman signal is obtained in a back scattering geometry from the (100) silicon surface. The phonon modes of silicon are classified as transverse optical phonons (TO) and longitudinal optical phonons (LO). The classification of a phonon in a TO or LO modes depends on the surface of porous silicon from which scattering data are observed. For instance, LO phonons occur for a back scattering from a (100) surface, and TO phonons are created from a (001) surface. This is the reason that some selected Raman modes have been observed by switching the scattering forms and measured surfaces $[19,20]$. As shown in Fig. 5, the LO phonon mode of porous silicon around at $522 \mathrm{~cm}^{-1}$ is seen for the back scattering from the (100) surface. A typical Raman profile of the observed mode of porous silicon is shown in Fig. 5 [21]. In Fig. 5 the Raman mode is slightly blue shifted about $0.8 \mathrm{~cm}^{-1}$ for the decrease in porosity as well as decrease in current density. The smaller shift $\left(0.8 \mathrm{~cm}^{-1}\right)$ is due to the presence of more homogeneity of the sample surfaces. It should be pointed out however, Raman features are strongly dependent on the fabrication parameters [15]. The Si-Si optical phonon mode intensity of porous silicon is considerably increased compared with crystalline silicon due to the surface area of porous silicon [22]. The results of this study support this observation as shown in Fig. 6.

However the intensity of the Raman band is also sensitive to the structure of the crystals and as a result, significant information can be obtained from intensity measurements. Generally visible light absorption is reduced in porous silicon compared to crystalline silicon. The formation of pores in silicon is an obvious reason for the increase in light penetration in porous silicon. This is also one of the reasons for the decrease in the layer density of porous silicon.

The Raman scattering intensity enhancement of porous silicon is observed because various current densities are utilized. In general, the Raman scattering intensity is proportional to the equilibrium population and inelastic scattering cross-section of phonons in this mode[ref]. The peak intensity of the Raman scattering signal from porous silicon is enhanced with the increase of the interaction area [22]. In comparison with crystalline silicon (substrate), the porous silicon surface possesses a dramatically different light absorption and scattering properties that could influence the inelastic scattering cross-section of phonon generated. With increasing surface area, the Raman scattering intensity of porous silicon also increases more than that of crystalline silicon.

The phonon confinement model may be used to tune the energy of the emitted light in nanoscale optical devices based on the nanoparticle size and shape. When the size of the particle reduces to the nanometer order, the wavefunction of optical phonons will no longer be a plane wave. The localization of wavefunction leads to a relaxation in the selection rule of wavevector conservation. Porous silicon typically forms in a columnar structure on the (100) face of the silicon substrate. This represents a typical two-dimensional quantum confinement. There are silicon nanostructures with a wide distribution of columnar diameters in which the length of the column is not confined [refs]. The average size of the confined structures can be determined by 
Preprint: Sangeetha Periasamy, Sasirekha Venkidusamy, Ragavendran Venkatesan, Jeyanthinath Mayandi, Joshua Pearce, Josefine Helene Selj,

Ramakrishnan Veerabahu. Micro-Raman Scattering of Nanoscale Silicon in Amorphous and Porous Silicon. Zeitschrift für Physikalische

Chemie, 231(9), pp. 1585-1598 (2017). DOI: https://doi.org/10.1515/zpch-2016-0961

the phonon confinement model. For nanocrystals, momentum conservation is no longer valid, since the optical phonons are localized.

According to the phonon confinement model (Equations $2 \& 3$ ), the estimated crystalline size L is approximately $40 \mathrm{~nm}$. This crystalline size is found to decrease when current density increases. Using this phonon confinement model it was determined that crystallite size in porous silicon is found to be in the range from 30 to $40 \mathrm{~nm}$, which is good agreement with the value obtained by Selji et al. [23] (Fig. 7). This leads to the formation of mesopores, whose diameter ranges from 2 to $50 \mathrm{~nm}$.

In contrast to expected red shift from the phonon confinement model, the Raman line shape of nanoscale porous silicon shows the considerable blue shift with respect to bulk silicon. This is due to the size dependent compressive stress in nanoscale porous silicon [24, 25]. The compressive stress is induced by the lattice mismatch with $\mathrm{Si}$ substrate at the base of porous silicon pillars. This could also be due to the numerous lattice mismatches introduced during the fabrication process of porous silicon and the differences in the elastic constant and the thermal expansion coefficients.

The two major factors which influence the magnitude of the Raman shift to stress coefficient are 1) the material parameters of the porous silicon and 2) the spatial orientation of the Raman detection. While determining the Raman shift due to the stress co-efficient the transversely isotropic properties of porous silicon have to be considered. It is also known that for the same porosity, each of the Raman shift to the stress co-efficient for the $x-y$ surface is the same as that for the $\mathrm{X}-\mathrm{Z}$ surface. The relation between the stress $(\sigma)$ and the Raman shift $(\Delta \omega)$ of porous silicon with respect to the porosities is given as follows [19]:

$$
\sigma=\left(232 \mathrm{R}_{\mathrm{po}}-190\right)(\Delta \omega)
$$

Where, $\mathrm{R}_{\mathrm{po}}$ denotes the porous silicon porosity and $\Delta \omega=\left(\omega_{\mathrm{ps}}-\omega_{\text {bulk }}\right)\left(\mathrm{in} \mathrm{cm}^{-1}\right), \omega_{\mathrm{ps}}$ is the Raman peak of $T_{2 g}$ mode of porous silicon under stress and $\omega_{\text {bulk }}$ is the Raman peak of $T_{2 g}$ mode of the stress - free silicon wafer. The value of compressive uniaxial stress is found to decrease when current density of porous silicon increases, which is shown in Table 2.

\section{Conclusion}

The micro-Raman spectra of various forms of nanoscale silicon in both amorphous silicon on quartz and porous silicon have been analyzed to determine the crystalline size of these materials. The silicon LO Raman line around at $521 \mathrm{~cm}^{-1}$ has been investigated in detail in order to get information on sample nanostructure. The $\mathrm{T}_{2 \mathrm{~g}}$ Raman peak of Si/quartz by PECVD gradually shifts from 504 to $494 \mathrm{~cm}^{-1}$ and the linewidth of the Raman scattering signal of these samples are broadened from 16 to $23 \mathrm{~cm}^{-1}$ with increasing rf deposition power from 15 to $50 \mathrm{~W}$, respectively. These results suggest that the samples are primarily amorphous in nature for deposition power at $15 \mathrm{~W}$ and $30 \mathrm{~W}$, but and the nanocrystallinity improves when deposition power is increased to $50 \mathrm{~W}$. The crystallite size of silicon of deposition power at $50 \mathrm{~W}$ was found to be $1 \mathrm{~nm}$ using phonon confinement model. In addition, the evolution on micro-Raman 
Preprint: Sangeetha Periasamy, Sasirekha Venkidusamy, Ragavendran Venkatesan, Jeyanthinath Mayandi, Joshua Pearce, Josefine Helene Selj,

Ramakrishnan Veerabahu. Micro-Raman Scattering of Nanoscale Silicon in Amorphous and Porous Silicon. Zeitschrift für Physikalische

Chemie, 231(9), pp. 1585-1598 (2017). DOI: https://doi.org/10.1515/zpch-2016-0961

scattering of porous silicon on silicon substrates formed by an electrochemical etching process in HF/ethanol solution was analyzed. The enhancement of Raman scattering intensity of porous silicon is observed as compared with crystalline silicon substrate. Unfortunately, the shift of Raman peak is small and thus does not provide much promise for optoelectronic application. However, this small shift can be used to deduce the crystalline size and stress of the porous silicon. The porous silicon hasnanocrystals in the range of $30-40 \mathrm{~nm}$ range as determined by the phonon confinement model. And also compressive stress of nanoscale porous silicon had been calculated. Finally we have obtained better results for nanostructured porous silicon compared with amorphous silicon on quartz substrate using micro-Raman spectroscopic technique.

\section{Acknowledgments}

This investigation was supported by the UGC-UPE programme. JM is thankful to the DST-SERB/F/1829/2012-2013 for partial support. V. R thanks the UGC, Government of India for providing micro Raman facilities under the UPE programme and PS is also thankful to UGC for the award of research fellowship under UPE programme. The authors also thank the Solar Energy Department, Institute for Energy Technology, Norway for providing samples.

\section{References:}

1. J-H. Lee, B-Y. Chu, B. Jung and S-C. Yang, Surface \& coatings technology, 201, 49614964 (2007).

2. A. M. Marmorstein, A. T. Voutsas and R. Solanki, Solid-State Electronics, 43, 305-313 (1999).

3. X. L. Wu, G. G. Siu, S. Tong, X. N. Liu, F. Yan, S. S. Jiang, X. K. Zhang and D. Feng, Appl. Phys. Lett, 69, 523-525 (1996).

4. S. Guha, P. Steiner and W. Lang, J. Appl. Phys, 79(11), 8664-8668 (1996).

5. J. Selj, A. Thogersen, S. E. Foss and E. S. Marstein, Thin solid films, 519, 2998-3001 (2011).

6. Ingrid De Wolf, IMEC, Kapeldreef 75, B-3001 Leuven, Belgium.

7. O. Tuzun, A. Slaoui, S. Roques, A. Focsa, F. Jomard and D. Ballutaud, Thin Solid Films, 517, 6358-6363 (2009).

8. N. P. Meshram, A. Kumbhar and R.O. Dusane, Thin Solid Films, doi:10.1016/j.tsf.2011.01.304.

9. D. Han, J. D. Lorentzen, J. Weinberg-Wolf, L. E. McNeil and Qi wang, J. Appl. Phys, 94, 2930-2936 (2003)

10. M.Benyoucel and M. Kuball, J. Appl. Phys, 89, 7903-7907 (2001).

11. Z. Iqbal and S. Veprek, J. Phys.C. Solid state Phys, 15, 377-392 (1982).

12. DING Wen-Ge, YUAN Jing, MENG Ling-Hai, WU Shu-Jie, YU Wei and FU Guang-Sheng, Commun. Theor. Phys, 55, 688-692(2011).

13. Yiqing Chen, Bo Peng and Bing Wang, J. Phys. Chem. C, 111, 5855-5858 (2007). 
Preprint: Sangeetha Periasamy, Sasirekha Venkidusamy, Ragavendran Venkatesan, Jeyanthinath Mayandi, Joshua Pearce, Josefine Helene Selj,

Ramakrishnan Veerabahu. Micro-Raman Scattering of Nanoscale Silicon in Amorphous and Porous Silicon. Zeitschrift für Physikalische

Chemie, 231(9), pp. 1585-1598 (2017). DOI: https://doi.org/10.1515/zpch-2016-0961

14. M. J. Konstantinovic, S. Bersier, X. Wang, M. Hayne, P. Lievens, R. E. Silverans, and V. V. Moshchalkov, Phy. Rev. B, 66, 161311(R) (2002).

15. S. Trusso, C. Vasi, M. Allegrini, F. Fuso, G. Pennelli, J. Vac. Sci. Technol. B, 17, 468-473 (1999).

16. Z. Sui, P. P. Leong, I. P. Herman, G. S. Higashi and H. Temkin, Appl. Phys. Lett, 60, 2086-2088 (1992).

17. Y. Duan, J. F. Kong and W. Z. Shen, J. Raman Spectrosc, J. Raman spectrosc, DOI 10.1002/jrs.3094 (2011).

18. T. D. Kang, H. Lee, S. J. Park, J. Jang and S. Lee, J. Appl. Phys, 92, 2467-2474 (2002).

19. Q. Li, W. Qui, H. Tan, J. Guo and Y. Kang, Optics and Lasers in Engineering, 48, 1119-1125 (2010).

20. LEI Zhen-kun, KANG Yi-Lan, HU Ming, QIU Yu, XU Han, NIU Hong-Pan, Chin.Phys. Lett, 21, 403-405 (2004).

21. S. K. Deb, N. Mathur, A. P. Roy, S. Banerjee and A. Sadesai, Solid State Commun, 101, 283-287 (1997).

22. Gong-Ru Lin, Yung-Hsiang Lin, Yi-Hao Pai and Fan-Shuen Meng, Opt. Express, 19, 597-605 (2011).

23. J. H. Selji, A. Thogersen, S. E. Foss and E. S. Marstein, Journal of applied physics, 107, 074904 (2010).

24. Sathyaprakash Sahoo, S. Dhara, S. Mahadevan and A. K. Arora, Journal of Nanoscience and Nanotechnology, 9, 5604-5607(4) (2009).

25. H. Kohno, T. Iwasaki, Y. Mita, S. Takeda, Journal of Applied Physics, 91, 32323235 (2002) 
Preprint: Sangeetha Periasamy, Sasirekha Venkidusamy, Ragavendran Venkatesan, Jeyanthinath Mayandi, Joshua Pearce, Josefine Helene Selj, Ramakrishnan Veerabahu. Micro-Raman Scattering of Nanoscale Silicon in Amorphous and Porous Silicon. Zeitschrift für Physikalische Chemie, 231(9), pp. 1585-1598 (2017). DOI: https://doi.org/10.1515/zpch-2016-0961

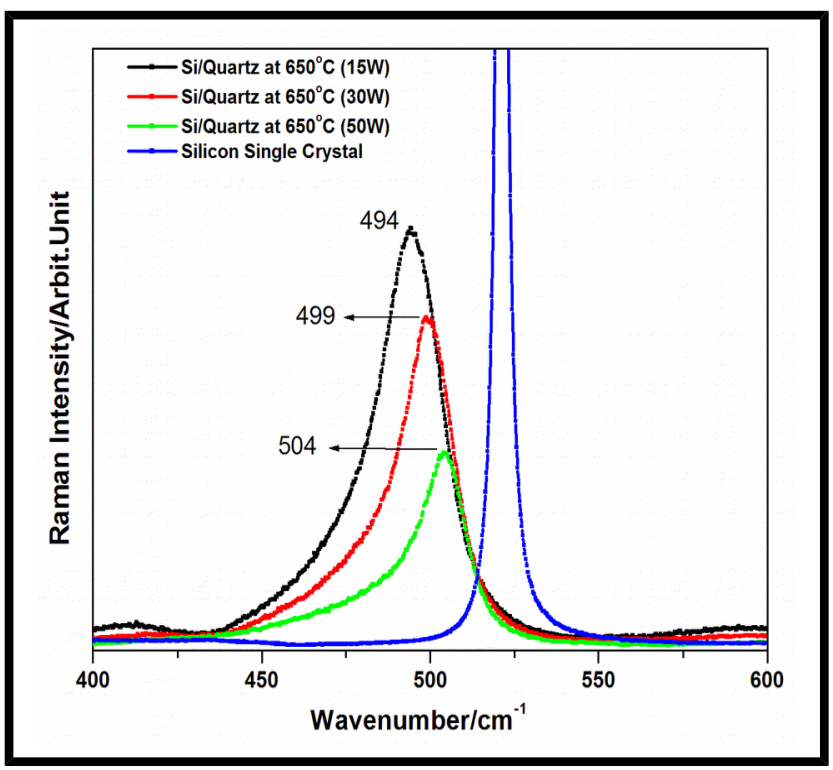

Fig. 1. Micro-Raman spectra of Si/Quartz with various deposition power (rf power) 
Preprint: Sangeetha Periasamy, Sasirekha Venkidusamy, Ragavendran Venkatesan, Jeyanthinath Mayandi, Joshua Pearce, Josefine Helene Selj, Ramakrishnan Veerabahu. Micro-Raman Scattering of Nanoscale Silicon in Amorphous and Porous Silicon. Zeitschrift für Physikalische Chemie, 231(9), pp. 1585-1598 (2017). DOI: https://doi.org/10.1515/zpch-2016-0961

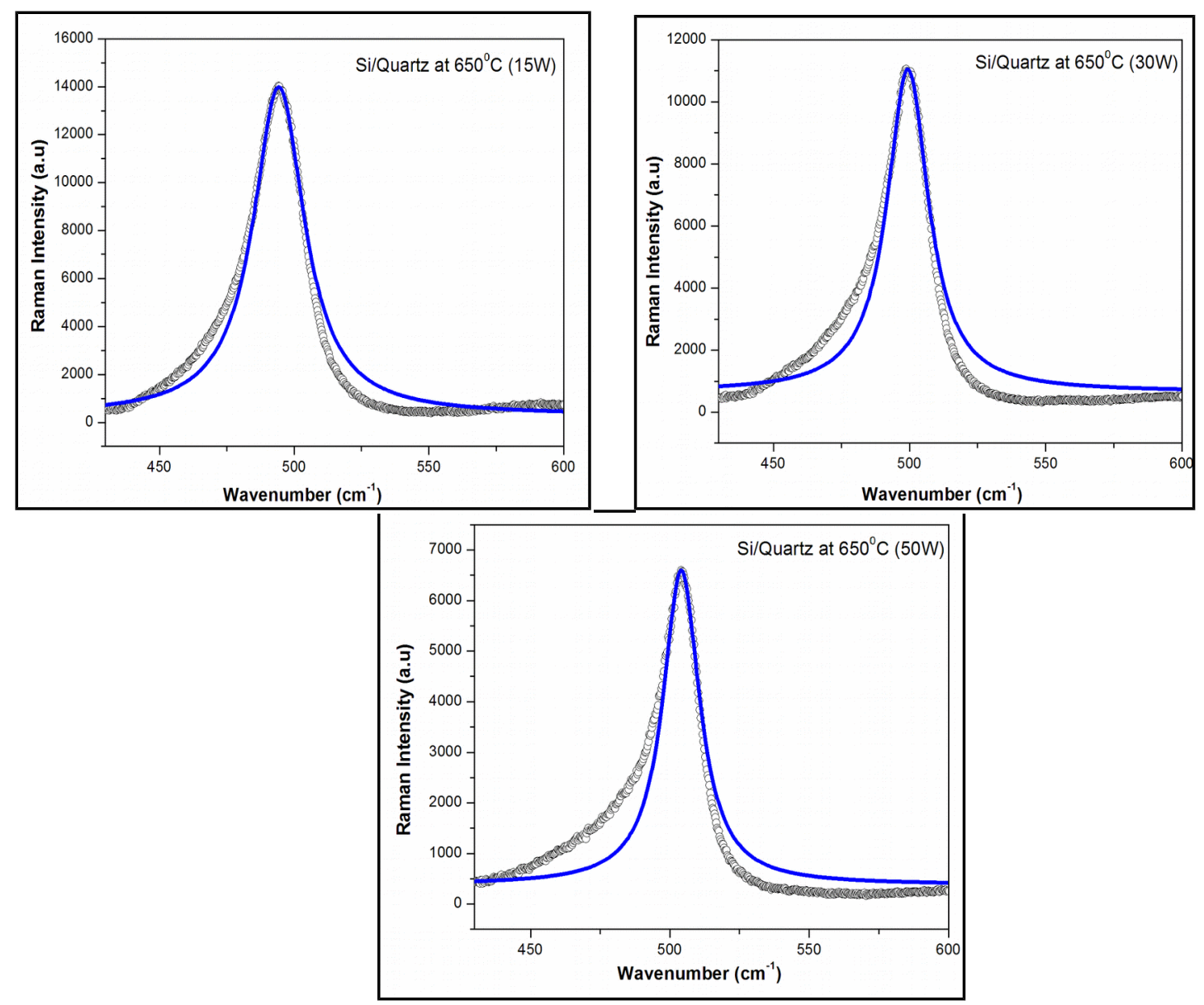

Fig. 2. Micro-Raman spectra of the $T_{2 g}$ phonon mode and the Lorentzian fit (solid line) for the $\mathrm{Si} / q u a r t z$ with various deposition power (rf power) 
Preprint: Sangeetha Periasamy, Sasirekha Venkidusamy, Ragavendran Venkatesan, Jeyanthinath Mayandi, Joshua Pearce, Josefine Helene Selj, Ramakrishnan Veerabahu. Micro-Raman Scattering of Nanoscale Silicon in Amorphous and Porous Silicon. Zeitschrift für Physikalische Chemie, 231(9), pp. 1585-1598 (2017). DOI: https://doi.org/10.1515/zpch-2016-0961

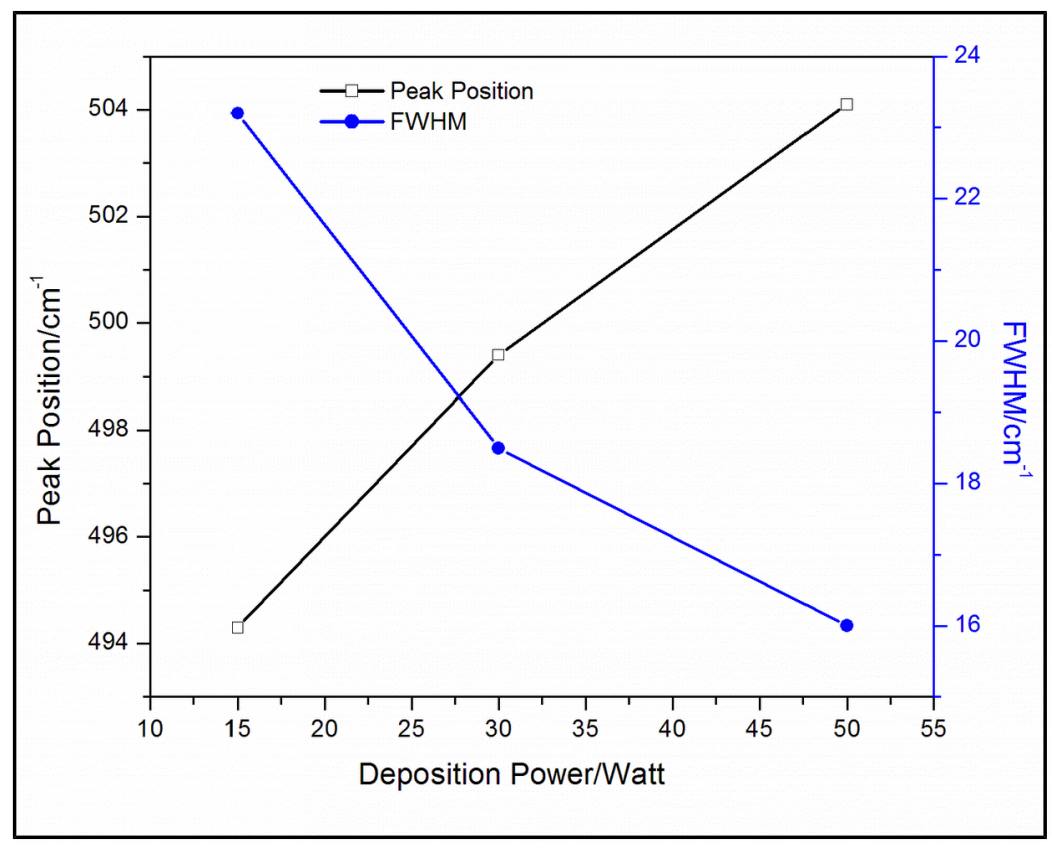

Fig. 3. The deposition power dependence of the FWHM and Raman peak position of Si/quartz 
Preprint: Sangeetha Periasamy, Sasirekha Venkidusamy, Ragavendran Venkatesan, Jeyanthinath Mayandi, Joshua Pearce, Josefine Helene Selj, Ramakrishnan Veerabahu. Micro-Raman Scattering of Nanoscale Silicon in Amorphous and Porous Silicon. Zeitschrift für Physikalische Chemie, 231(9), pp. 1585-1598 (2017). DOI: https://doi.org/10.1515/zpch-2016-0961

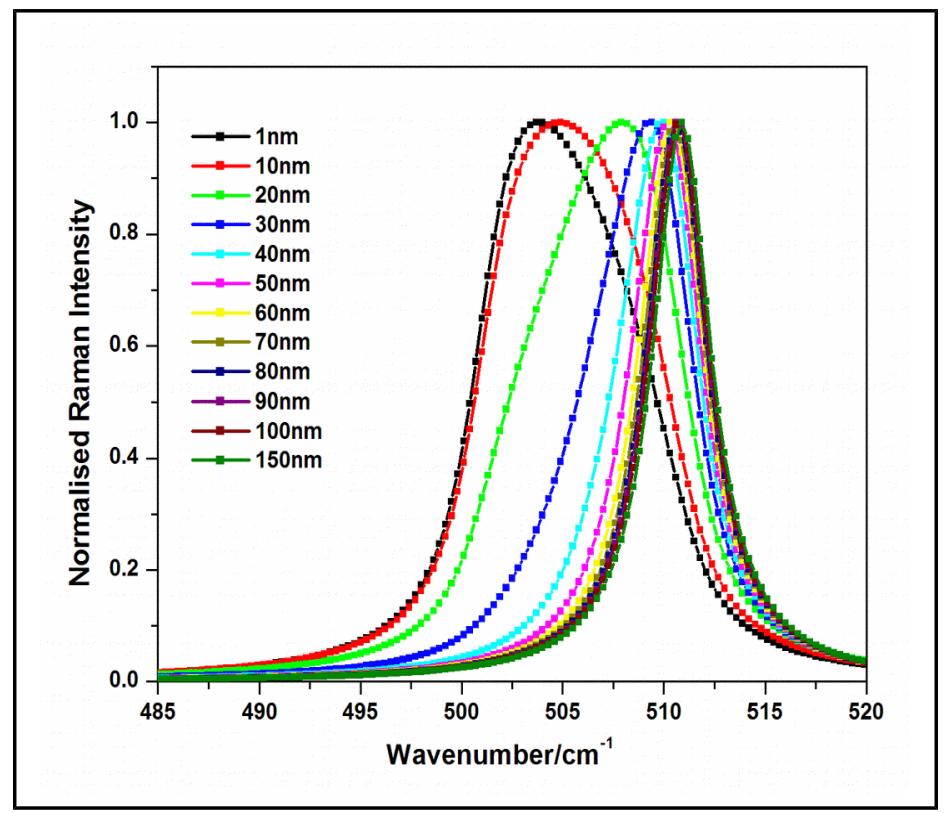

Fig. 4. The calculated Raman spectra of $T_{2 g}$ mode of Si with different grain sizes from Phonon confinement model. 
Preprint: Sangeetha Periasamy, Sasirekha Venkidusamy, Ragavendran Venkatesan, Jeyanthinath Mayandi, Joshua Pearce, Josefine Helene Selj, Ramakrishnan Veerabahu. Micro-Raman Scattering of Nanoscale Silicon in Amorphous and Porous Silicon. Zeitschrift für Physikalische Chemie, 231(9), pp. 1585-1598 (2017). DOI: https://doi.org/10.1515/zpch-2016-0961

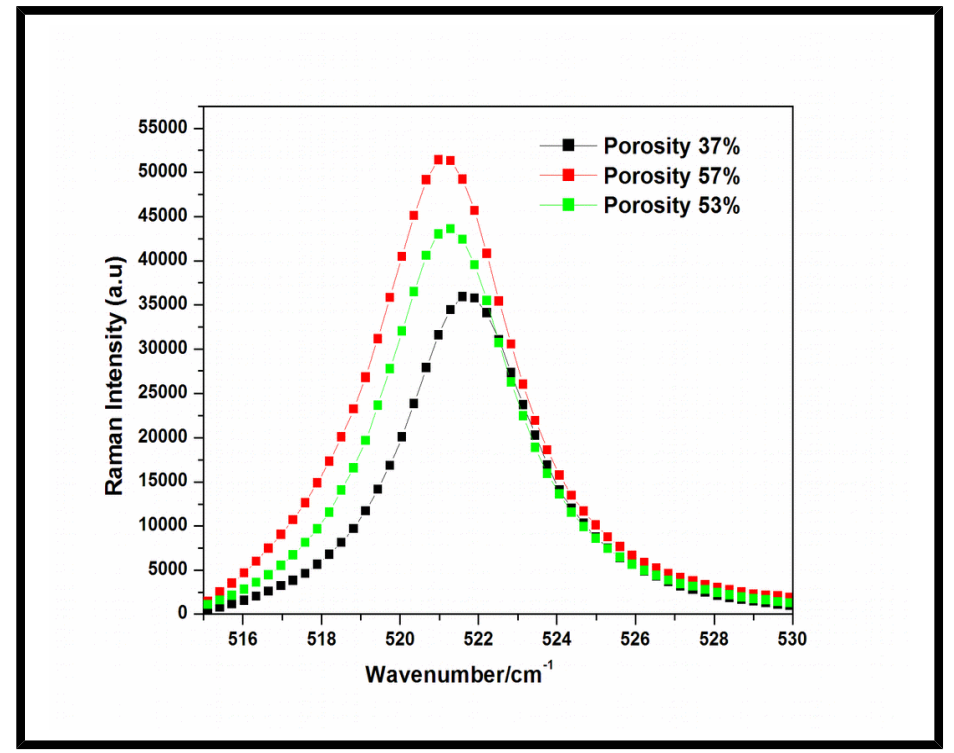

Fig. 5. Micro-Raman spectra of $T_{2 g}$ mode of porous silicon on silicon with different porosity
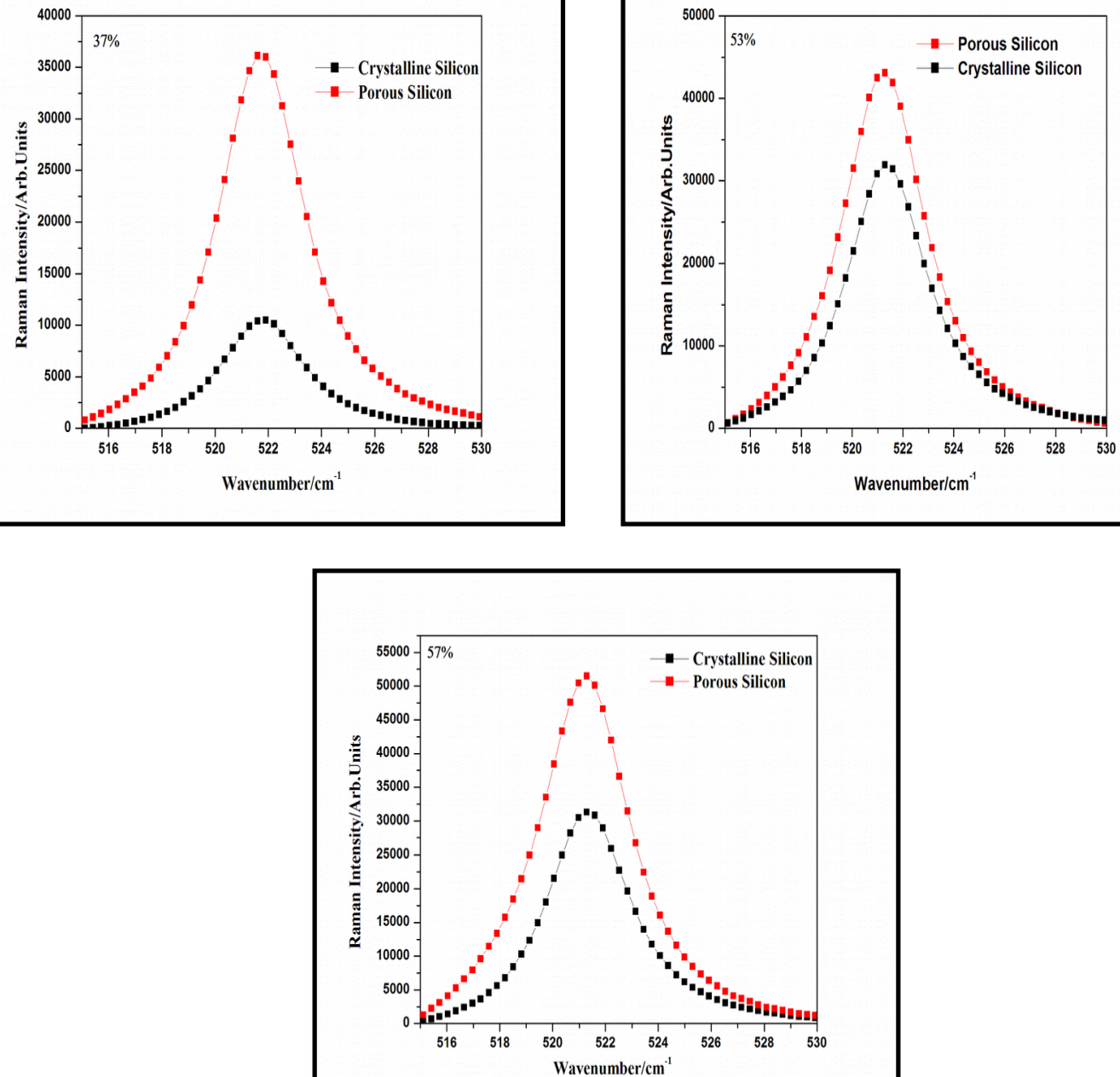
Preprint: Sangeetha Periasamy, Sasirekha Venkidusamy, Ragavendran Venkatesan, Jeyanthinath Mayandi, Joshua Pearce, Josefine Helene Selj, Ramakrishnan Veerabahu. Micro-Raman Scattering of Nanoscale Silicon in Amorphous and Porous Silicon. Zeitschrift für Physikalische Chemie, 231(9), pp. 1585-1598 (2017). DOI: https://doi.org/10.1515/zpch-2016-0961

Fig. 6. Micro-Raman spectral comparison of porous-silicon with crystalline-silicon for different porosity.
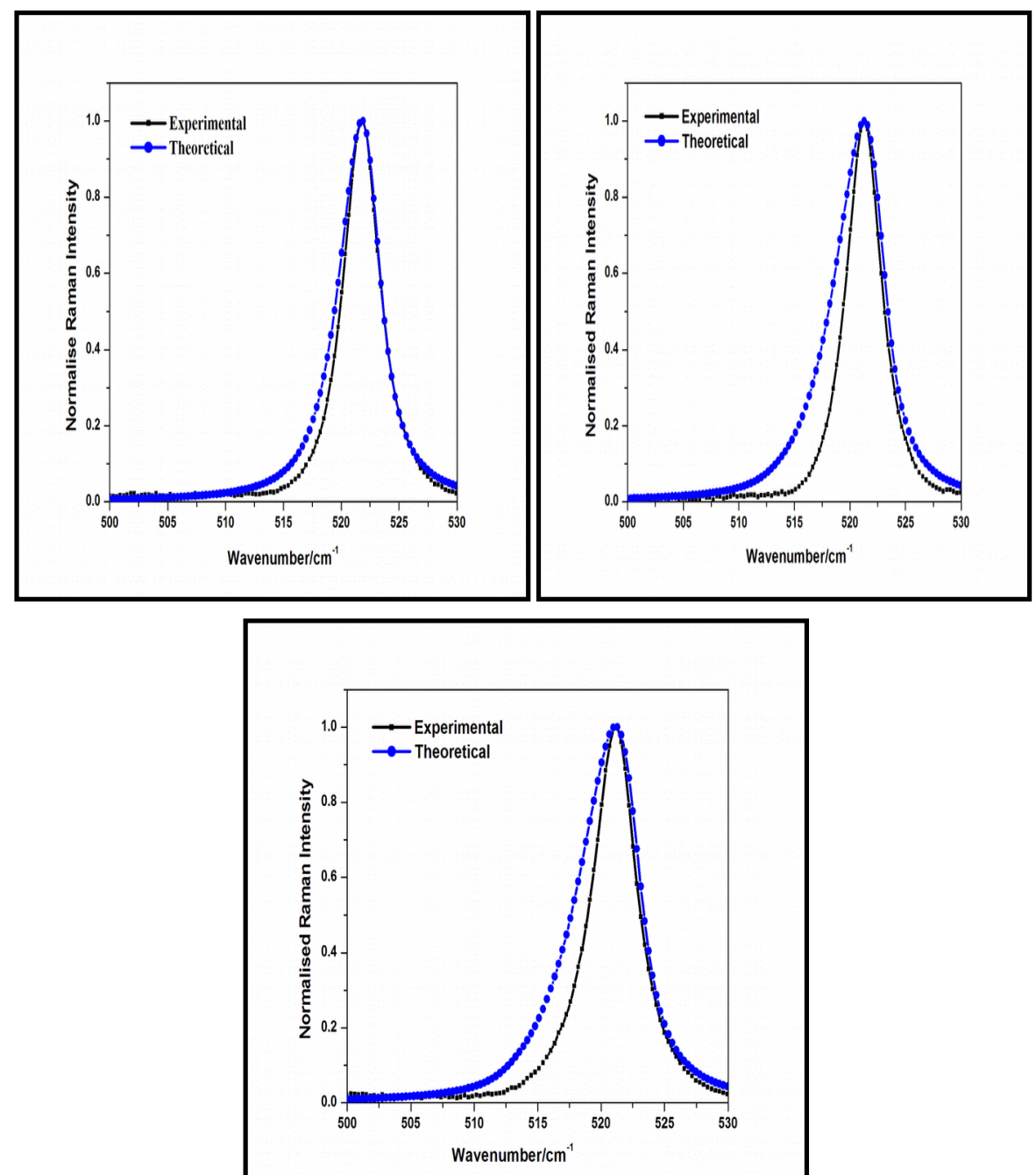

Fig. 7. Micro Raman spectra of $T_{2 g}$ mode of porous $\mathrm{Si}$ (black line) fitted with theoretical model (blue line) (a) porosity $37 \%$ (b) porosity $53 \%$ (c) porosity $57 \%$ 
Preprint: Sangeetha Periasamy, Sasirekha Venkidusamy, Ragavendran Venkatesan, Jeyanthinath Mayandi, Joshua Pearce, Josefine Helene Selj, Ramakrishnan Veerabahu. Micro-Raman Scattering of Nanoscale Silicon in Amorphous and Porous Silicon. Zeitschrift für Physikalische Chemie, 231(9), pp. 1585-1598 (2017). DOI: https://doi.org/10.1515/zpch-2016-0961

Table 1. Lorentzian fit parameters of $\mathbf{T}_{2 \mathrm{~g}}$ mode of Si/Quartz

\begin{tabular}{cccc}
\hline Sample Details & FWHM $\left(\mathbf{c m}^{-1}\right)$ & Peak position $\left(\mathbf{c m}^{-1}\right)$ & Area (a.u) \\
\hline $\mathbf{1 5} \mathbf{W}$ at $\mathbf{6 5 0} 0^{\circ} \mathrm{C}$ & 23.2 & 494 & 498804 \\
$\mathbf{3 0} \mathbf{W}$ at $\mathbf{6 5 0} \mathbf{C}$ & 18.5 & 499 & 299981 \\
$\mathbf{5 0} \mathbf{W}$ at $\mathbf{6 5 0}$ & & & \\
& 16 & 504 & 156348 \\
\hline
\end{tabular}

Table 2. Experimental and Theoretical results of porous silicon with different etching parameters

\begin{tabular}{ccccc}
\hline $\begin{array}{c}\text { Current } \\
\text { density }\end{array}$ & Porosity & $\begin{array}{c}\text { Raman peak }\left(\mathbf{c m}^{-\mathbf{1}}\right) \\
\text { (measured) }\end{array}$ & $\begin{array}{c}\text { Crystallite } \\
\text { size (nm) }\end{array}$ & $\begin{array}{c}\text { Stress } \\
(\mathbf{G P a})\end{array}$ \\
\hline $\mathbf{1 0} \mathbf{~} \mathbf{A} / \mathbf{c m}^{2}$ & $37 \%$ & 521.8 & 40 & 0.08 \\
$\mathbf{5 0} \mathbf{~} \mathbf{A} / \mathbf{c m}^{2}$ & $53 \%$ & 521.2 & 32 & 0.03 \\
$\mathbf{1 2 5} \mathbf{~} \mathbf{m A} / \mathbf{c m}^{2}$ & $57 \%$ & 521.2 & 30 & 0.01 \\
\hline
\end{tabular}

